Article

\title{
Exogenous Therapeutics of Microrna-29a Attenuates Development of Hepatic Fibrosis in Cholestatic Animal Model through Regulation of Phosphoinositide 3-Kinase p85 Alpha
}

\author{
Ya-Ling Yang ${ }^{1,+}$, Feng-Sheng Wang ${ }^{2,+}$, Hung-Yu Lin ${ }^{3,4, * \mathbb{D}}$ and Ying-Hsien Huang ${ }^{5, *}$ (I) \\ 1 Department of Anesthesiology, Kaohsiung Chang Gung Memorial Hospital and Chang Gung University \\ College of Medicine, Kaohsiung 833, Taiwan; inr453@cgmh.org.tw \\ 2 Core Laboratory for Phenomics \& Diagnostics, Department of Medical Research, Kaohsiung Chang Gung \\ Memorial Hospital and Chang Gung University College of Medicine, Kaohsiung 833, Taiwan; \\ wangfs@ms33.hinet.net \\ 3 Department of Internal Medicine, Kaohsiung Chang Gung Memorial Hospital and Chang Gung University \\ College of Medicine, Kaohsiung 833, Taiwan \\ 4 Center for Mitochondrial Research and Medicine, Kaohsiung Chang Gung Memorial Hospital and Chang \\ Gung University College of Medicine, Kaohsiung 833, Taiwan \\ 5 Department of Pediatrics, Kaohsiung Chang Gung Memorial Hospital and Chang Gung University College \\ of Medicine, Kaohsiung 833, Taiwan \\ * Correspondence: linhungyu700218@gmail.com (H.-Y.L.); yhhuang123@yahoo.com.tw (Y.-H.H.) \\ + These authors contributed equally to this work.
}

Received: 21 April 2020; Accepted: 19 May 2020; Published: 21 May 2020

check for updates

\begin{abstract}
Recent studies have found that microRNA-29a (miR-29a) levels are significantly lower in fibrotic livers, as shown with human liver cirrhosis. Such downregulation influences the activation of hepatic stellate cells (HSC). Phosphoinositide 3-kinase p85 alpha (PI3KP85 $\alpha$ ) is implicated in the regulation of proteostasis mitochondrial integrity and unfolded protein response (UPR) and apoptosis in hepatocytes. This study aimed to investigate the potential therapeutic role of miR-29a in a murine bile duct ligation (BDL)-cholestatic injury and liver fibrosis model. Mice were assigned to four groups: sham, BDL, BDL + scramble miRs, and BDL + miR-29a-mimic. Liver fibrosis and inflammation were assessed by histological staining and mRNA/protein expression of representative markers. Exogenous therapeutics of miR-29a in BDL-stressed mice significantly attenuated glutamic oxaloacetic transaminase (GOT)/glutamic-pyruvic transaminase (GPT) and liver fibrosis, and caused a significant downregulation in markers related to inflammation (IL-1 $\beta$ ), fibrogenesis (TGF- $\beta 1, \alpha$-SMA, and COL1 $\alpha 1$ ), autophagy (p62 and LC3B II), mitochondrial unfolded protein response (UPR ${ }^{\mathrm{mt}}$; C/EBP homologous protein (CHOP), heat shock protein 60 (HSP60), and Lon protease-1 (LONP1, a mitochondrial protease), and PI3KP85 $\alpha$ within the liver tissue. An in vitro luciferase reporter assay further confirmed that miR-29a mimic directly targets mRNA $3^{\prime}$ untranslated region (UTR) of PI3KP85 $\alpha$ to suppress its expression in HepG2 cell line. Our data provide new insights that therapeutic miR-29a improves cholestasis-induced hepatic inflammation and fibrosis and proteotstasis via blocking PI3KP85 $\alpha$, highlighting the potential of miR-29a targeted therapy for liver injury.
\end{abstract}

Keywords: microRNA-29a; hepatitis; fibrosis; therapeutics; PI3KP85 $\alpha$; mitochondrial unfolded protein response; proteostasis 


\section{Introduction}

Mounting evidence has highlighted persistent liver injury results in liver fibrosis, which involves various cell types. Following a persistent liver injury, hepatic stellate cells (HSCs) are activated and experience morphologic and functional trans-differentiation into contractile myofibroblastic cells [1]. Moreover, activated HSCs are responsible for secretion of profibrogenic mediators, transforming growth factor- $\beta$ (TGF- $\beta$ ), and generates extracellular matrix (ECM) proteins that can worsen the wound-healing process, including collagen types I/III, fibronectin, and laminin [2]. MicroRNAs (miRNAs) are a class of highly conserved short noncoding RNAs that regulate gene expression at a post-transcriptional level. A considerable decrease of miR-29 family (a to c) in humans with liver cirrhosis and a hepatic fibrosis animal model induced by the administration of hepatotoxin has been reported. Furthermore, their role in affecting HSC activation has been demonstrated. TGF- $\beta$ can be secreted by hepatocytes, Kupffer cells, and sinusoidal endothelial cells, functioning as an activator for HSC activation [3]. Mechanistically, TGF- $\beta 1$ causes HSC to activate, transdifferentiate, and secrete ECM through the downregulation of microRNA-29a (miR-29a) [4,5]. Of note, the overexpression of miR-29a in murine HSC has been shown to suppress fibrogenic genes such as collagen- $1 \alpha 1$ (COL1 $\alpha 1)$ and collagen-4 $\alpha 1$ [4-6], by directly targeting these extracellular matrix genes' mRNA. However, evidence of whether miR-29a level is implicated in the pathogenesis of acute cholestasis is still scarce and whether exogenous miR-29a exerts therapeutic potential is yet to be investigated.

Recent lines of study suggest that phosphoinositide 3-kinase p85 alpha (PI3KP85 $\alpha$ ) is involved in the mechanisms in response to aberrant proteotoxic stress in the liver or hepatocytes. Proteotoxic stress occurring in liver diseases such as non-alcoholic fatty liver disease (NAFLD), nonalcoholic steatohepatitis (NASH), and fibrosis can induce ER stress, autophagy, as well as mitochondrial unfolded protein response $\left(\mathrm{UPR}^{\mathrm{mt}}\right)$, which is a transcriptional response induced by mitochondrial dysfunction and relayed by a retrograde mitochondria-to-nucleus crosstalk $[7,8]$. Activation of $\mathrm{UPR}^{\mathrm{mt}}$ requires phosphorylated eukaryotic translation initiation factor 2 subunit 1 (eIF $2 \alpha$ ) to reduce global protein synthesis while boosting the expression of transcription factors, including C/EBP homologous protein (CHOP), ATF4, and ATF5. These transcription factors are involved in activating genes functioning to repair the proteotoxic stress, including chaperones and proteases [7]. Furthermore, activation of CHOP plays a central role in regulating inflammation, fibrogenesis, and autophagic flux in the pathogenesis of hepatitis and liver fibrosis $[9,10]$. In this regard, PI3KP85 $\alpha$ is required to relay experimental ER stress and CHOP activation in tunicamycin-or alcohol-stressed liver [11,12]. However, whether miR-29a modifies PI3KP85 $\alpha$ expression to exert a therapeutic effect in liver fibrosis is yet to be clarified.

In recent years, our research team has been dedicated to exploring the molecular mechanism of miR-29a in the pathogenesis of liver fibrosis [1,13-21]. We have already demonstrated that genetic overexpression of miR-29a exerts a protective effect against hepatic damage and fibrosis via a number of pathways in the cholestatic mouse model [1,15-20]. However, evidence of whether miR-29a can exert a therapeutic effect on cholestatic liver damage is still scarce. As for our previous studies and other literature, we hypothesize that the therapeutic exogenous administration of miR-29a via tall-vein injection may mitigate murine bile duct ligation (BDL)-induced cholestatic liver injury and fibrosis through direct targeting PI3KP $85 \alpha$.

\section{Results}

\subsection{Exogenous miR-29a Injection Significantly Reduces Liver Injury and Fibrosis in the Context of BDL}

To develop in vivo delivery via intravenous administration, a polymer-based material in vivo-jetPEI ${ }^{\circledR}$ to condense miR-29a in nanoscale particles was utilized. We firstly performed a pilot experiment that included no-treated (NT) and miR-29a mimic administration at 0,1 or $5 \mathrm{nmole} / 30 \mathrm{~g}$, showing that 1 nomle caused relatively effective increase in hepatic miR-29a level ( $p=0.06$, Figure S1) and was chosen as administration dose thereafter. Mice were allocated to four groups: sham-operated control, BDL, BDL + scramble, and BDL + miR-29a-mimic. A seven-day experimental flow chart is 
shown as Figure 1A. BDL per se had no effect on miR-29a expression in the liver, compared with that in sham, while exogenous miR-29a administration increased two to three times compared with other experimental groups ( $p<0.05$, Figure 1B). BDL, BDL + scramble, and BDL + miR-29a presented a decrease in the body weight and liver-to-body percentage, compared with sham group at day 7 (Table 1). BDL + miR-29a showed an increase in body weight gain compared to BDL, but not to BDL + scramble. Both BDL + scramble and BDL + miR-29a showed an increase in liver-to-body ratio (Table 1). Masson trichrome staining used to determine hepatic fibrosis showed that BDL group exhibited more collagen-matrix-accumulated blue staining around the portal area in liver specimens than that of BDL surgery mice, but not in the sham group $(p<0.05$, Figure 1C-D). This histopathology of fibrosis has been significantly reduced in BDL + miR-29a $(p<0.05$, compared with BDL and BDL + scramble; Figure 1C-D). Furthermore, alpha-smooth muscle actin ( $\alpha$-SMA) protein expression, which denotes a marker for HSC activation and hepatic fibrosis, was decreased in BDL-miR29a, compared with that in $\operatorname{BDL}(p<0.05$, Figure 1E). These results indicate that exogenous miR-29a injection via tail veil exerts therapeutic effect in ameliorating hepatic inflammation and fibrosis in cholestatic liver.

A
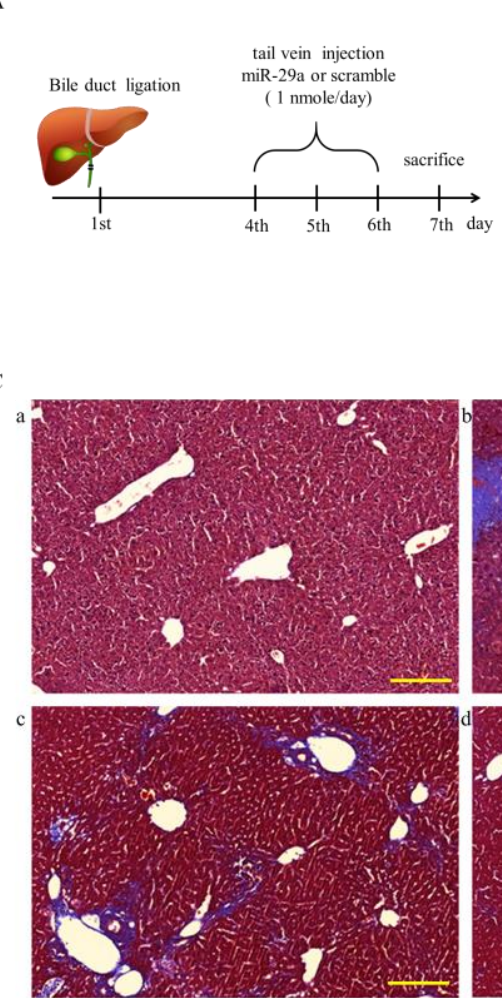

B
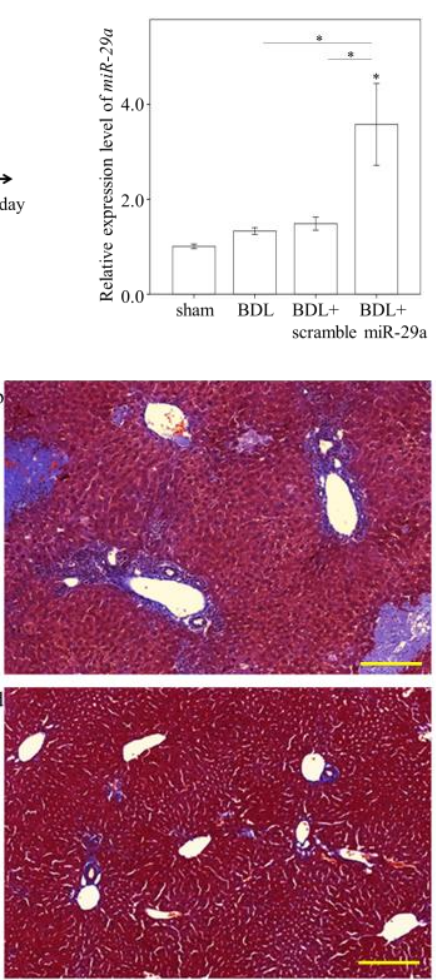

D

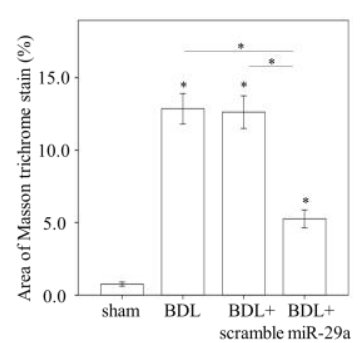

$\mathrm{E}$

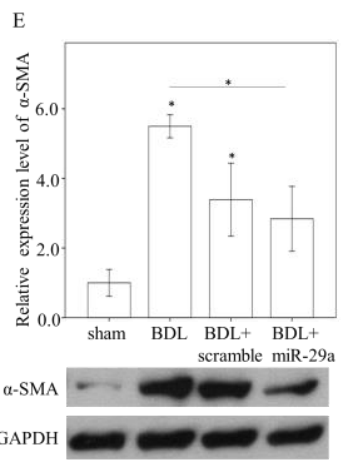

Figure 1. Exogenous miR-29a injection significantly reduces liver fibrosis in the context of BDL. (A) Experimental procedure. (B) quantitative real-time PCR (qRT-PCR) results of miR-29a levels in liver specimens. $N=6-13$. (C) Representative image of Masson trichrome staining. a: sham, b: BDL, c: $\mathrm{BDL}+$ scramble, $\mathrm{d}$ : BDL+miR-29a. Blue stain indicates collagen matrix accumulation. Scale bar, 200 $\mu \mathrm{m}(\mathrm{D})$ quantification results of Masson trichrome staining. Positive staining area (\%) was quantified using ImageJ. $\mathrm{N}=6-7$. (E) Representative blotting image and densitometric results of $\alpha$-SMA protein expression. $\mathrm{N}=6$ for each group. Histogram data are expressed as mean $\pm \mathrm{SE} .{ }^{*} p<0.05$ between the groups. Sham, sham surgery only. BDL, bile duct ligation operation only. BDL + scramble, mice received exogenous scramble injection after BDL. BDL + miR-29a, mice received exogenous miR-29a injection after BDL. $\alpha$-SMA, alpha-smooth muscle actin. 
Table 1. Anthropometric measurements of the animals.

\begin{tabular}{|c|c|c|c|c|c|}
\hline Parameter & Time Point & Sham & BDL & $\begin{array}{c}\text { BDL + } \\
\text { Scramble }\end{array}$ & BDL+miR-29a \\
\hline \multirow[t]{2}{*}{ Body Weight (g) } & Day 0 & $23.25 \pm 0.51$ & $23.26 \pm 0.29$ & $24.23 \pm 0.32$ & $24.46 \pm 0.35$ \\
\hline & Day 7 & $23.93 \pm 0.53$ & $18.72 \pm 0.35^{a}$ & $18.77 \pm 0.23^{a}$ & $18.82 \pm 0.27^{a}$ \\
\hline Body Weight Gain (\%) & Day 7 & $2.9 \pm 0.52$ & $-19.48 \pm 1.17^{a}$ & $-22.52 \pm 0.96^{a}$ & $-22.97 \pm 1.39^{a, b}$ \\
\hline Liver Weight (g) & Day 7 & $1.19 \pm 0.07$ & $1.36 \pm 0.04^{a}$ & $1.21 \pm 0.06$ & $1.22 \pm 0.05$ \\
\hline Liver/Body Weight (\%) & Day 7 & $4.94 \pm 0.21$ & $7.25 \pm 0.13^{a}$ & $6.44 \pm 0.26^{a, b}$ & $6.49 \pm 0.18^{a, b}$ \\
\hline
\end{tabular}

Data collected from seven to ten mice each group expressed as mean $\pm \mathrm{SE} ;{ }^{a}, p<0.05$ versus sham; ${ }^{b}, p<0.05$ versus BDL. BDL: bile duct ligation.

\subsection{Exogenous Administration of miR-29a via Tail Vin Injection Significantly Restores the Markers Assessing} Hepatic Inflammation and Fibrosis

BDL induced hepatic inflammation, as evidenced by an increase in serum GOT, GPT, and total bilirubin level, $(p<0.05$, Figure 2A-D). BDL + miR-29a presented a lower GOT/GPT level than BDL + scramble $(p<0.05$, Figure 2A-B), indicating hepatoprotective effect of miR-29a. However, as BDL + scramble showed a higher GOT/GPT value than BDL group $(p<0.05$, Figure $2 \mathrm{~A}, \mathrm{~B})$, we deduced that an off-target effect derived from exogenous small RNA, which can perturb innate immune response [22], might be involved. On the other hand, BDL, the BDL + scramble, and BDL + miR-29 group showed a lower GOT/GTP ratio than sham group $(p<0.05$, Figure $2 \mathrm{C})$. Then, we confirmed the expression level of genes corresponding to histological and biochemical manifestations by using qRT-PCR. The mRNA level of inflammatory marker Il1b, and fibrogenic markers colla1 and $t g f b 1$, was increased in BDL group, compared with other groups (all $p<0.05$, Figure 2D-F), and significantly decreased in $\mathrm{BDL}+$ miR-29a group (all $p<0.05$, Figure $2 \mathrm{E}-\mathrm{G}$ ).
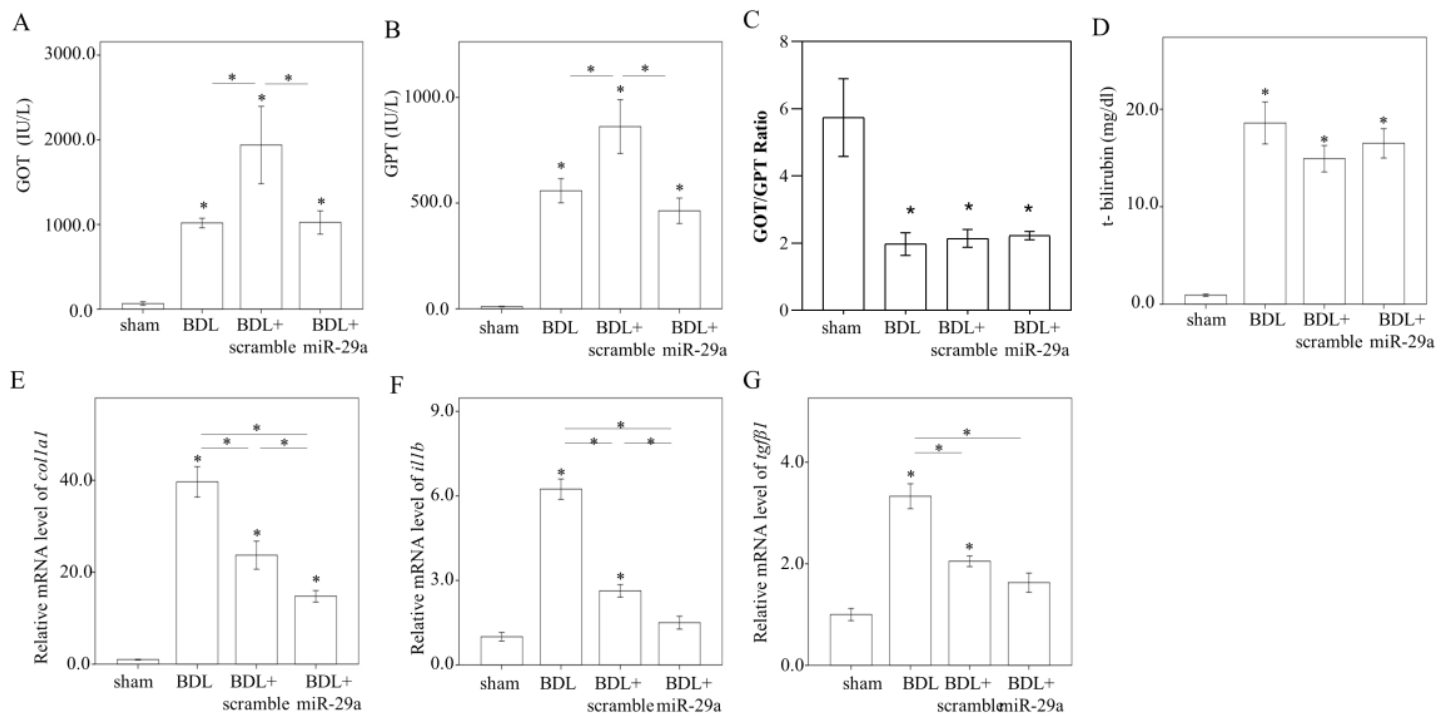

Figure 2. Exogenous miR-29a injection significantly reverses the markers assessing hepatic inflammation and fibrosis. (A-D) Serum GOT, GPT, GOT/GPT ratio, and total bilirubin. mRNA expression level of (E) colla1, (F) Il1b, and (G) tgfb1. $\beta$-actin level is used as the normalization control. $\mathrm{N}=5-9$ was used for each group. Data are expressed as mean \pm SE. ${ }^{*} p<0.05$ between the groups. Sham, a sham surgery only. BDL, a bile duct ligation operation only. BDL + scramble, mice received exogenous scramble injection after BDL. BDL + miR-29a, mice received exogenous miR-29a injection after BDL. GOT: glutamic oxaloacetic transaminase. GPT: glutamic-pyruvic transaminase. 
2.3. Exogenous miR-29a Injection Significantly Reduced PI3KP85 $\alpha$, as well as Molecules Associated with $U P R^{\text {mt }}$ and Autophagy in Colestatic Livers

Given that PI3KP85 $\alpha$ was shown to be central to relay proteostatic signaling in stressed liver [11,12], and is predicted as a target of miR-29a from bioinformatic database ( $w w w . m i r b a s e . o r g)$, we hypothesize that PI3KP85 $\alpha$ may be involved in the mechanism mediating the effect of miR-29a. As shown in Figure 3A, western blot analysis showed that PI3KP85 $\alpha$ was induced in BDL, compared with sham group, and was reduced in $\mathrm{BDL}+\mathrm{miR}-29$ a group $(p<0.05)$. Likewise, $\mathrm{CHOP}$, the putative downstream of PI3KP85 $\alpha$, was increased in BDL, compared with sham group, while reduced in BDL + miR-29a group ( $p<0.05$, Figure 3B). UPR ${ }^{\mathrm{mt}}$ effector HSP60 and LONP1, presented corresponding expression manner in line with CHOP $(p<0.05$, Figure 3C-D). Other proteostatic stress makers p62 and LC3B II were also increased in BDL, compared with sham, while decreased in BDL + miR-29a $(p<0.05$, Figure $3 \mathrm{E}, \mathrm{F})$. These results suggest that miR-29a administration mediates inhibition of PI3KP $85 \alpha$ to alleviate proteotoxic stress in cholestatic liver.

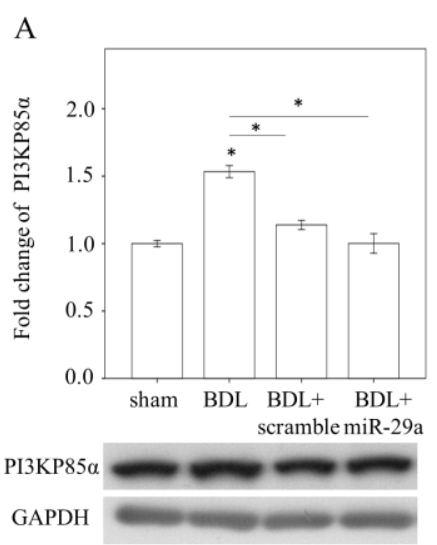

$\mathrm{D}$

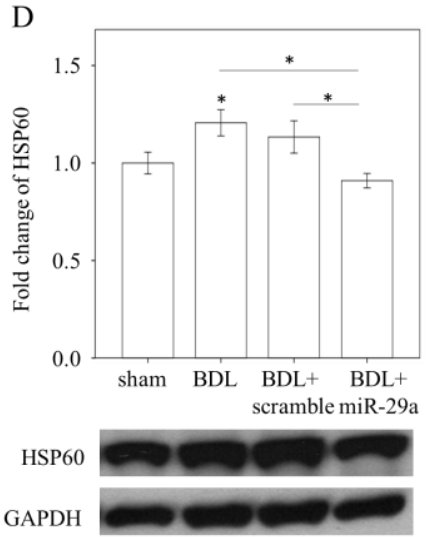

B
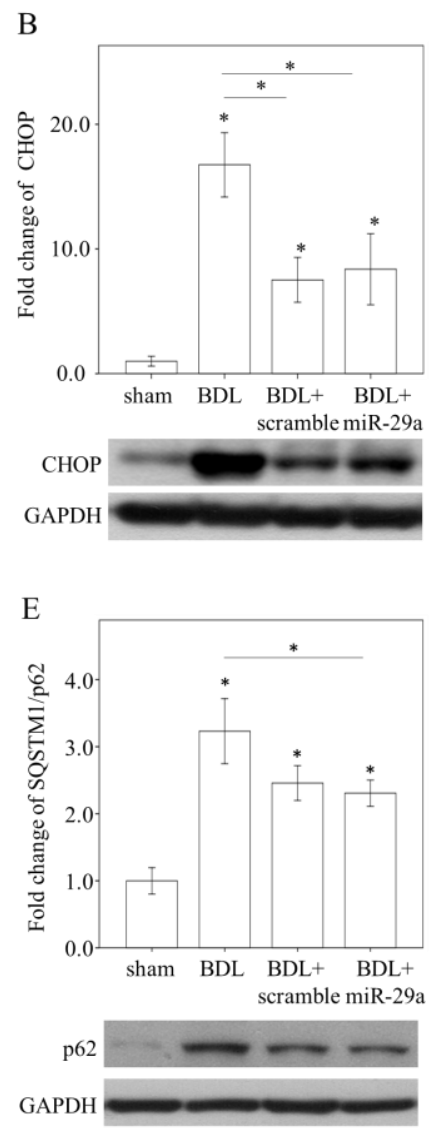
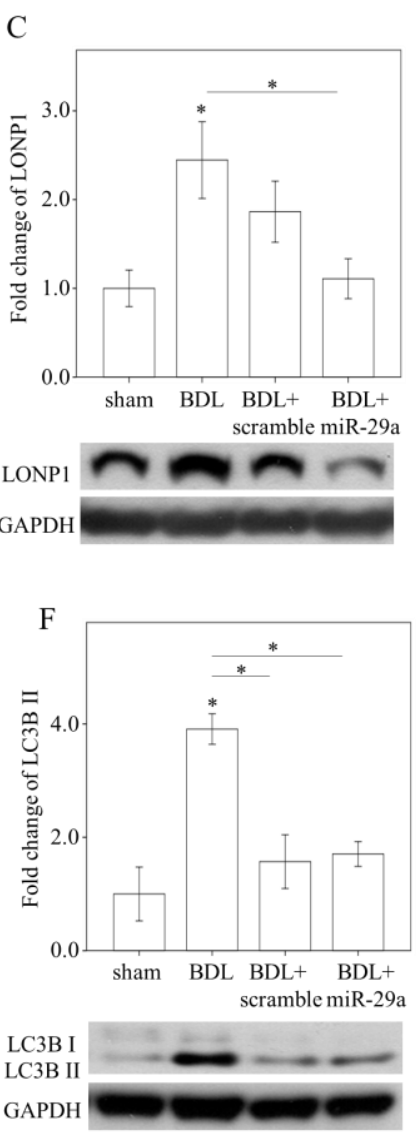

Figure 3. Effect of miR-29a treatment on protein expression of PI3Kp85 $\alpha$ and proteostatic molecules in the liver. Representative image of western blot and densitometric results of PI3Kp85 $\alpha$ (A), CHOP (B), LONP1 (C) HSP60 (D), SQSTM1/p62 (E), and LC3B II (F), in mice liver. Data collected from N = 5-6 per group are expressed as mean \pm SE. ${ }^{*} p<0.05$ between the groups. Sham, a sham surgery only. BDL, a bile duct ligation operation only. BDL + scramble, mice received exogenous scramble injection after BDL. BDL + miR-29a, mice received exogenous injection of miR-29a mimic after BDL.

\section{4. miR-29a Acts to Suppress PI3Kp85 $\alpha$ Expression via Directly Targeting its $3^{\prime} U T R$}

To verify the suppressive activity of miR-29a on mRNA $3^{\prime}$ untranslated region (UTR) of PI3KP85 $\alpha$, we conducted in vitro luciferase reporter assay in human liver hepatocellular carcinoma HepG2 cells harboring PI3Kp85 $\alpha-3^{\prime}$ UTR or PI3Kp85 $\alpha-3^{\prime}$ UTR mutant (Mut) luciferase reporter construct. MiR-29a 
mimic or control scramble sequence was transfected into HepG2 cells. As shown in Figure 4, miR-29a mimic significantly reduced the luciferase activity in cells harboring wildtype PI3Kp $85 \alpha-3^{\prime} \mathrm{UTR}$, compared with miR-scramble, while the miR-29a mimic-derived suppressive effect diminished in cells harboring the PI3Kp $85 \alpha-3^{\prime}$ UTR Mut (all $p<0.05$, Figure 4). This result confirms that miR-29a inhibited the expression of PI3Kp $85 \alpha$ by targeting its $3^{\prime} \mathrm{UTR}$.
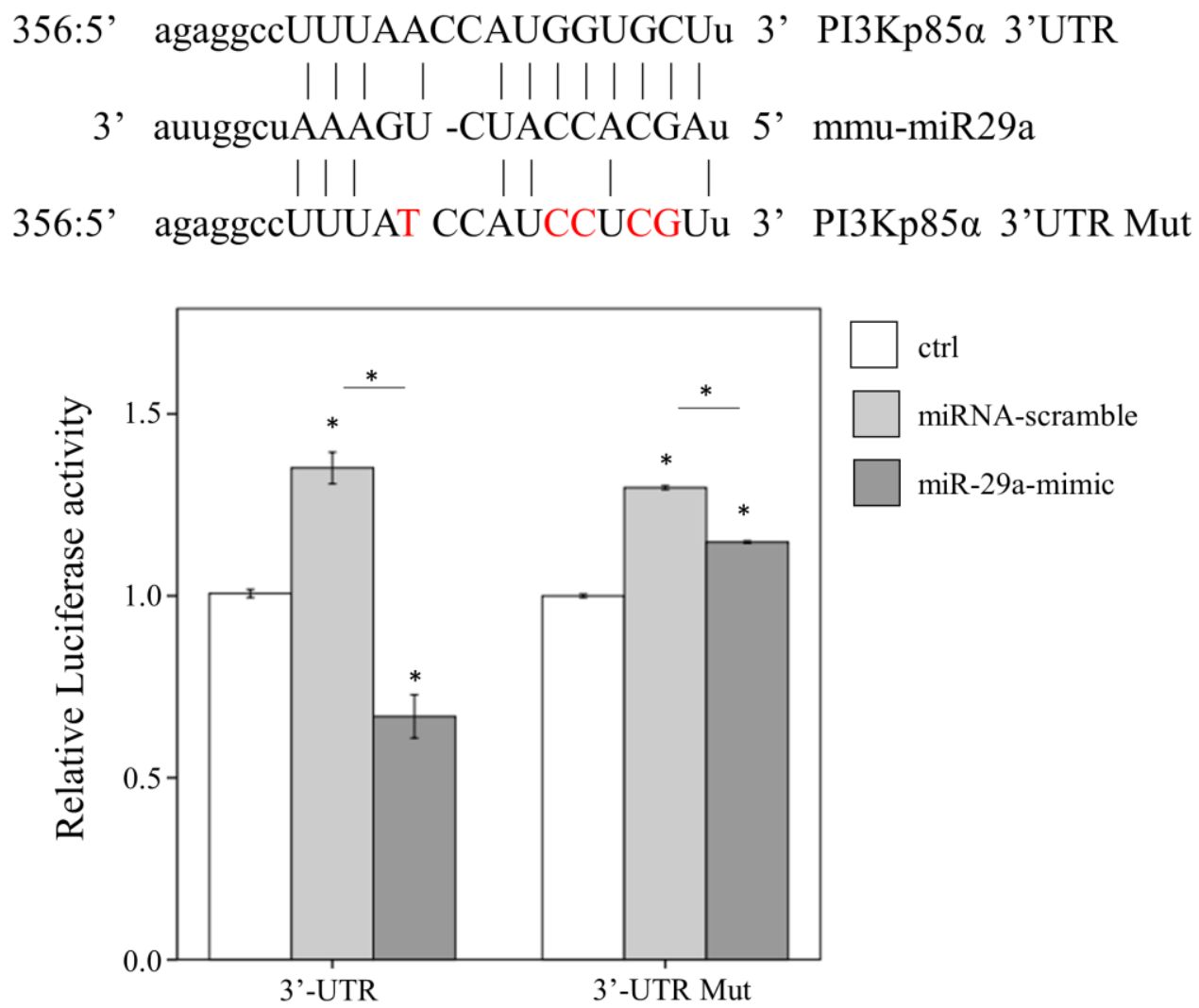

Figure 4. Interaction of miR-29a with the $3^{\prime}$ UTR of PI3Kp $85 \alpha$ by luciferase assay. Shown above is the sequence information and mutual binding status of PI3Kp85-3'UTR, mmu-miR29a, and PI3Kp85-3'UTR Mut. The nucleotides in red indicate mismatching sites. HepG2 cells were firstly transfected with PI3Kp85 $\alpha-3^{\prime}$ UTR or PI3Kp85 $\alpha-3^{\prime}$ UTR mutant luciferase reporter plasmid, then treated with control medium (ctrl), miRNA-scramble, or miR-29a mimic, and finally lysed to detect the luciferase signal. Three independent experiments $(\mathrm{N}=3)$ with at least triplicate for each experiment were conducted. Data expressed as mean \pm SE. * indicates a $p<0.05$ between the groups. mmu-miR29a, mouse-origin miR-29a. ctrl, control. Mut, mutant. UTR, untranslated region.

\section{Discussion}

In this study, we demonstrated that intravenous miR-29a administration exerts protective effect on hepatic inflammation and fibrosis in cholestatic mouse liver. In addition, we revealed that miR-29a targets and suppresses PI3KP85 $\alpha$ expression along with downregulation of proteostatic molecules, including CHOP, HSP60, LONP1, p62, and LC3B II. A proposed model of miR-29a-relayed pathway is depicted in Figure 5. 


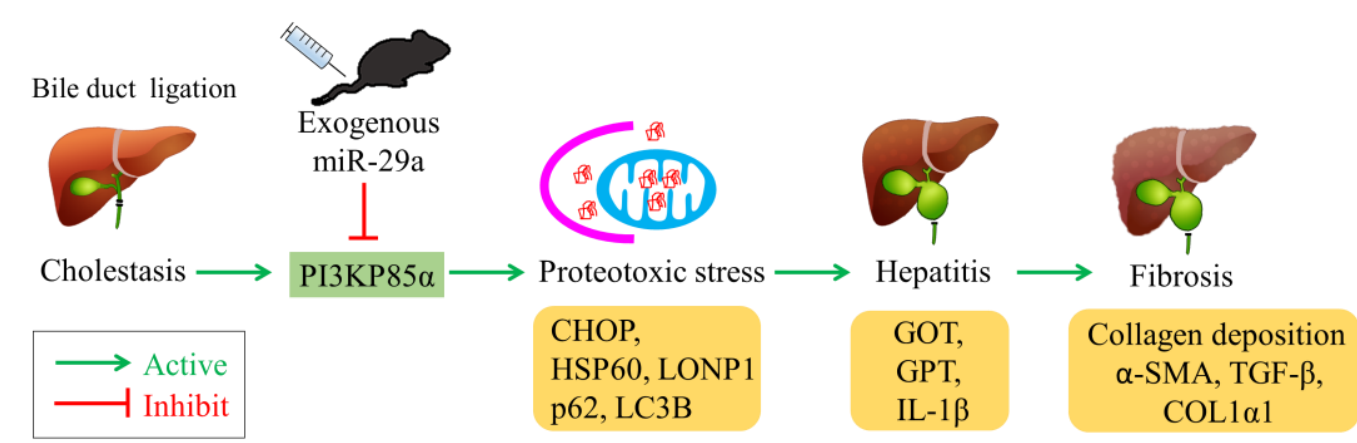

Figure 5. Proposed model of miR-29a-relayed pathway in the treatment of cholestasis. Exogenous miR-29a ameliorates cholestasis-induced proteotoxic stress, hepatitis, and liver fibrosis by suppressing PI3K85 $\alpha$.

A decrease of miR-29a abundance has been reported in the liver of patients with cirrhosis and that of mouse treated with chemical hepatotoxin $[4,23]$, which take years and weeks to form pathological manifestation, respectively. In contrast, the present study demonstrates that BDL insult, which sustains over a span of three days, has no effect on hepatic miR-29a level, indicating miR-29a expression manner in the context of acute damage differs from that in relatively chronic situation. Nevertheless, our previous study demonstrates that mice harboring overexpressed miR-29a present ameliorated BDL-induced liver fibrosis [1,19]. Of note, this study further exhibits that exogenous administration of miR-29a exerts hepatic protection, highlighting the potential of miR-29a in new drug development.

In our previous studies, mice with overexpression of miR-29a present alleviated liver damage in the context of cholestasis induced by BDL $[1,15,16,18,19]$. Nevertheless, these results are not sufficient to extrapolate that miR-29a possesses therapeutic effect, because a C57BL/6 mouse harbors overexpressed miR-29a from birth and is distinct from scenario using treatment approach. In the present study, we sought to condense miR-29a by a polymer-based material and conducted in vivo delivery via intravenous administration and demonstrated its therapeutic effect in counteracting hepatic inflammation and fibrosis (Figures 1 and 2). Although information regarding exogenous miR-29a administration in cholestatic liver is limited, Matsumoto et al. have shown hepatoprotective effect of intravenous delivery of miR-29a in a chronic liver fibrosis model induced by $\mathrm{CCl}_{4}$ [23], in line with our observation.

Off-target activity of noncoding RNA such as miR, siRNA and shRNA, through activation of some types of Toll-like receptors, can complicate the interpretation of phenotypic results and may lead to unexpected toxicity to the cells [22]. In our study, scramble miR administration causes an increase in serum GOT and GPT level (BDL vs. BDL + scramble, Figure 1B), raising concern about off-target effect. However, scramble miR is nondetrimental in some parameters, including having no influence on liver fibrosis (BDL vs. BDL + scramble, Figure 1C), a decrease in liver-to-body weight (BDL vs. BDL + scramble, Table 1), and reduced level in markers related to inflammation and fibrogenesis (BDL vs. $\mathrm{BDL}+$ scramble, Figure 2B-D). Importantly, miR-29a mimic per se has no detrimental effect on all parameters. Nevertheless, further study is warranted and should take safety, and toxicity issue of miR-based approach, into consideration.

Our previous study has demonstrated that the ER stress is involved in the pathogenesis of BDL-induced liver fibrosis, and its provocation can be alleviated in mice harboring miR-29a transgene [18]. Of note, the mutual interaction between mitochondria and ER regulates proteostasis and cellular stress by the activity of mitochondrial-localizing proteins and interorganellar communication $[24,25]$. In the present study, induced CHOP, which functions as a key mediator of both ER stress and UPR ${ }^{\mathrm{mt}}$, is suppressed by miR-29a mimic, implying that not only mitochondria but also ER stress may be affected in this scenario. Further study is warranted to elaborate on the role of miR-29a on ER stress and ER-mitochondria crosstalk in the context of cholestasis. 
Several lines of study have suggested a deteriorative role of PI3KP85 $\alpha$ in the development of liver fibrosis. In vitro studies demonstrated that pharmacological inhibition of PI3KP $85 \alpha$ exerts an antifibrogenic effect in human HSC and liver tissue [26,27]. Son et al. presented that PI3K inhibition by adenovirus-based delivery attenuates in vitro fibrogenic activity in HSC and in vivo hepatic fibrosis, while fails to ameliorate hepatic inflammation [28], indicating specific inhibition to PI3K likely not effective in liver injury. In fact, potential hepatoxicity of PI3K inhibitor has been noted [29]. In this regard, our study demonstrates that intravenous administration of miR-29a exerts inhibitory effect of PI3KP85 $\alpha$, and significant improvement in both hepatic inflammation and fibrosis. As such, miR-based exogenous approach appears to be superior. Nevertheless, the exact mechanism needs to be clarified in future study.

The consequence of PI3K inhibition in the hepatocytes results in suppression of CHOP activity, leading to impaired mitochondrial homeostasis [12]. Furthermore, CHOP along with HSP60 and LONP1 constituting $\mathrm{UPR}^{\mathrm{mt}}$ are central to modulating mitochondrial proteostasis [7]. Abrogation of $\mathrm{CHOP}$ was shown to mitigate hepatic inflammation and fibrosis as well as proteotoxic response $[9,30]$. In the context of NASH/NAFLD, provoked autophagic activity is suppressed by CHOP silencing or deletion [10]. Herein, our study demonstrates that miR-29a-supresses PI3KP85a to exert inhibitory effect on $\mathrm{CHOP}$, which may contribute to a lessened mitochondrial proteotoxic status, as evidenced by reduced HSP60 and LONP1 as well as p62 and LC3B II (Figure 3). Although the molecular mechanism that controls miR-29a/PI3KP85a/CHOP involvement in the stressed liver is still not clear, a recent study suggests an emerging role of miRs in preserving the hepatic mitochondrial proteostasis [31].

\section{Materials and Methods}

\subsection{Ethics Statement}

Our animal protocol was reviewed and approved by the Institutional Animal Care and Use Committee (IACUC) of Chang Gung Memorial Hospital (19 December 2017; Approval number: 2017091801). We purchased C57BL/6, 7-week-old mice from BioLASCO Taiwan Co., Ltd. (Taipei, Taiwan) All animals were housed in an animal facility at $22{ }^{\circ} \mathrm{C}$, with a relative humidity of $55 \%$, in a 12 $\mathrm{h}$ light $/ 12 \mathrm{~h}$ dark cycle, with food and sterile tap water available ad libitum.

\subsection{Animal Model and Experimental Protocol}

Six to eight mice were used for all our experiments. The operation procedure of common bile duct ligation or sham control was described in a previous study [1]. MiR-29a mimic (UAGCACCAUCUGAAAUCGGUUA; \#C-300504-07-0050, Horizon Discovery) and scramble control sequence (UCACAACCUCCUAGAAAGAGUAGA; \#CN-001000-01-50, respectively, purchased from Horizon Discovery) used for the purpose of in vivo delivery were dissolved in $200 \mu \mathrm{L}$ of in vivo-jetPEI ${ }^{\circledR}$ reagent (201-50G, Polyplus-transfection) in accordance with the manufacturer's standard protocol. Surgical operation was conducted at the 1st day. From the 4th to 6th day, the BDL group was allocated to two groups of tail vein injection with scrambled (BDL + scramble, 1 nmole/30 g) or miR-29a-mimic (BDL + miR-29a, 1 nmole/30 g), one shot per day, three shots in total. Anthropometric measurements were conducted at the beginning and end of the study. All the mice were euthanized 1 week postoperatively. Liver tissues were dissected, snap-frozen, and processed, to isolate total RNA and proteins. All specimens were stored at $-80^{\circ} \mathrm{C}$ until the biochemical analysis was carried out.

\subsection{Histological Analysis}

Fresh livers were fixed in in 10\% formaldehyde and embedded in paraffin. Five micrometer sections were subjected to Masson trichrome stain (Poly Scientific, Bay Shore, NY, USA) in accordance with the manufacturer's standard protocol, except for the $90 \mathrm{~min}$ incubation of aniline blue-solution I. The prolonged incubation was an optimized condition in our laboratory. The quantification of staining 
signal was analyzed by independent color channel of ImageJ (version 1.48, Wayne Rasband, National Institutes of Health, Rockville, MD, USA).

\subsection{Quantitative Real-Time PCR ( $q R T-P C R$ )}

Total RNA was extracted by using TRIzol ${ }^{\circledR}$ reagent (Invitrogen, Carlsbad, CA, USA) from liver tissue and used to generate cDNA with an oligodeoxynucleotide primer (oligo dT15) according to the manufacturer's protocol (Promega, Madison, WI). MicroRNA Isolation Kits (BioChain Institute, Inc, Hayward, CA, USA) was used for to isolate total microRNA. The qPCR reaction of COL1 $\alpha 1$, IL- $1 \beta$, and tgf- $\beta$ as well as normalization control glyceraldehyde 3-phosphate dehydrogenase (GAPDF)was conducted with 2X SYBR Green PCR Master Mix (Roche Molecular Systems, Inc., Pleasanton, CA, USA) on LightCycler $480^{\circledR}$ (Roche). Each PCR reaction included $5 \mu \mathrm{M}$ forward and reverse primers and the cDNA product diluted at $8 \times$ in a total reaction volume of $10 \mu \mathrm{L}$. For qPCR reaction of COL1 $\alpha 1, \mathrm{IL}-1 \beta$ and $\operatorname{tg} f-\beta$, an initial amplification was done with a denaturation step at $95^{\circ} \mathrm{C}$ for $10 \mathrm{~min}$, followed by 45 cycles of denaturation at $95^{\circ} \mathrm{C}$ for $30 \mathrm{~s}$, primer annealing at $62{ }^{\circ} \mathrm{C}$ for $15 \mathrm{~s}$, and primer extension at $72{ }^{\circ} \mathrm{C}$ for $25 \mathrm{~s}$ followed by melting curve analysis. The primer sequences were as follows. COL1 $\alpha 1$ : Forward sequence $5^{\prime}$-CTGGCAAGAATGGCGAC-3, reverse sequence 5'-CCCTGGAGACCAGAGAAG-3'; IL-1 $\beta$ : forward sequence 5'-GAGGACATGAGCACCTTCTTT-3', reverse sequence $5^{\prime}$-GCCTGTAGTGCAGTTGTCTAA- $3^{\prime}$; TGF- $\beta 1$ : forward sequence $5^{\prime}$-GTGGAC CGCAACAACGCCATCT-3', reverse sequence 5'-GCAATGGGGGTT CGGGCACT-3'; GAPDF: forward sequence $5^{\prime}$-GCACAGTCAAGGCCGAGAAT-3', reverse sequence $5^{\prime}$-GCCTTCTCCATG GTGGTG-3'. The PCR efficiency of COL1 $\alpha 1$, IL-1 $\beta$, TGF- $\beta 1$, and GAPDF is $1.913,1.978,1.981$, and 1.964, respectively (Figure S2). For detection of miR-29a expression, predesigned primer/probes for miR-29a (\#002112, ThermoFisher) and normalization control sno202 (\#001232, ThermoFisher) were used.

\subsection{Western Blotting}

Forty microgram proteins extracted from the liver was separated in 8-15\%SDS-PAGE, then transferred onto PVDF membrane and incubated with primary antibodies at $4{ }^{\circ} \mathrm{C}$ overnight. The primary antibodies included PI3Kp85 $\alpha$ (1:5000; 60225-1 lg, PROTEINTECH, IL, USA), CHOP (1:1000; \#2895, cell signaling, MA, USA), SQSTM1/p62 (1:5000; GTX111393, GeneTex, CA, USA), HSP60 (1:5000; sc-1052, Santa Cruz, CA, USA), LONP1 (1:2000; 15440-1-AP, Proteintech, IL, USA), LC3B II (1:5000; \#2775, cell signaling, MA, USA), and $\alpha$-SMA (1:1000; ab5694, abcam, Cambridge, UK). GAPDH (1:100,000; 60004-1 $\mathrm{lg}$, PROTEINTECH, IL, USA) was used for probing protein loading control. After washing twice with TBST solution, PVDF membrane was incubated with secondary antibodies such as horseradish peroxidase-coupled antirabbit immunoglobulin-G antibodies (1:5000; NEF812001EA, PerkinElmer, MA, USA) or HRP antimouse immunoglobulin-G antibodies (1:10,000; NEF822001, PerkinElmer) at room temperature for $1 \mathrm{~h}$. The blots were developed with an ECL Western blotting detection and analysis system (Amersham Pharmacia Biotech, Uppsala, Sweden) and exposed them to film. The signals were quantified by using Quantity One ${ }^{\circledR}$ 1-D analysis software (Bio-Rad Laboratories).

\subsection{Luciferase Reporter Assay}

The wild type pMIR-PI3Kp $85 \alpha$ luciferase plasmid was constructed by cloning mouse PI3Kp $85 \alpha-3^{\prime}$ UTR sequence into the pMIR-REPORT ${ }^{\mathrm{TM}}$ miRNA Expression Reporter Vector (Applied Biosystems, Foster City, CA, USA), while the pMIR-PI3Kp85 $\alpha$-Mut luciferase plasmid was substituted with five mismatched sites (Figure 4). The plasmids were purified using EasyPrep EndoFree Maxi Plasmid Extraction Kit (BIOTOOLS, Ltd., New Taipei, Taiwan). $9 \times 10^{5}$ HepG2 cells were seeded at a $6 \mathrm{~cm}$ culture dish. After $24 \mathrm{~h}, 3 \mu \mathrm{g}$ of pMIR-PI3Kp $85 \alpha$ luciferase plasmid or pMIR-PI3Kp85 $\alpha$-Mut plasmid was introduced by TurboFect reagent (Thermo Fisher Scientific, Rockford, IL, USA). After another $24 \mathrm{~h}, 25 \mathrm{nM}$ of miR-29a precursor (mimic-miR-29a, GE Healthcare Dharmacon, IN, USA) or miR control sequence (GE Healthcare Dharmacon) were introduced by using Lipofectamine ${ }^{\mathrm{TM}}$ RNAiMAX 
Transfection Reagent (Invitrogen) as per the manufacturer's standard protocol. After incubated for $24 \mathrm{~h}$ at $37^{\circ} \mathrm{C}$, the cells were lysed for the detection of luciferase signal with Neolite Reporter Gene Assay System (PerkinElmer, Waltham, MA, USA).

\subsection{Statistical Analysis}

Data collected from at least three independent experiments are expressed as mean \pm standard error. Statistical significance between groups was analyzed by one-way analysis of variance (ANOVA), followed by the least significant difference (LSD) test for post-hoc testing. Significant level was set at $p<0.05$. Graph drawing and statistical analysis were conducted with IBM SPSS Statistics V22.0.

\section{Conclusions}

Our findings demonstrate that intravenous administration of miR-29a renders hepatoprotection to cholestatic liver and alleviation of proteotoxic loading by targeting PI3KP85 $\alpha$. Therefore, our results suggest that miR-29a mimic could serve as a possible therapeutic tool to improve the treatment of liver inflammation and fibrosis.

Supplementary Materials: The following are available online at http://www.mdpi.com/1422-0067/21/10/3636/s1, Figure S1. Dose optimization of miR-29a mimic, Figure S2. PCR efficiency of col1 $\alpha 1, i l-1 \beta$, tgf- $\beta 1$, and gapdh.

Author Contributions: All authors substantially contributed to this work. Y.-H.H. designed the concept of the present study and supervised the study. Y.-L.Y. and F.-S.W. performed experiments, collected samples, and acquired the data. H.-Y.L., F.-S.W., and Y.-L.Y. analyzed and interpreted the data. H.-Y.L. and F.-S.W. drafted the article. Y.-H.H. critically revised the article for important intellectual content. All authors read and approved the final manuscript.

Funding: This study was supported by grants from the National Health Research Institute (NHRI-EX107-10736SI); Ministry of Science and Technology, Taiwan (106-2314-B-182A-141 -MY3 and 108-2811-B-182A-509); and Chang Gung Memorial Hospital, Taiwan (CMRPG8I0491, 8I0492 and 8G1342). However, these organizations had no part in the study design, data collection and analysis, publication decisions, or preparation of the manuscript.

Acknowledgments: The authors would like to thank Yuan-Ting Chuang and Chia-Ling Wu for assisting with this article.

Conflicts of Interest: The authors hereby declare no conflicts of interest about this article.

\section{References}

1. Tiao, M.M.; Wang, F.S.; Huang, L.T.; Chuang, J.H.; Kuo, H.C.; Yang, Y.L.; Huang, Y.H. MicroRNA-29a protects against acute liver injury in a mouse model of obstructive jaundice via inhibition of the extrinsic apoptosis pathway. Apoptosis 2014, 19, 30-41. [CrossRef] [PubMed]

2. Friedman, S.L. Hepatic stellate cells: Protean, multifunctional, and enigmatic cells of the liver. Physiol. Rev. 2008, 88, 125-172. [CrossRef] [PubMed]

3. Gressner, A.M.; Weiskirchen, R.; Breitkopf, K.; Dooley, S. Roles of TGF-beta in hepatic fibrosis. Front. Biosci. J. Virtual Libr. 2002, 7, d793-d807. [CrossRef]

4. Roderburg, C.; Urban, G.W.; Bettermann, K.; Vucur, M.; Zimmermann, H.; Schmidt, S.; Janssen, J.; Koppe, C.; Knolle, P.; Castoldi, M.; et al. Micro-RNA profiling reveals a role for miR-29 in human and murine liver fibrosis. Hepatology 2011, 53, 209-218. [CrossRef] [PubMed]

5. Bandyopadhyay, S.; Friedman, R.C.; Marquez, R.T.; Keck, K.; Kong, B.; Icardi, M.S.; Brown, K.E.; Burge, C.B.; Schmidt, W.N.; Wang, Y.; et al. Hepatitis $C$ virus infection and hepatic stellate cell activation downregulate miR-29: miR-29 overexpression reduces hepatitis C viral abundance in culture. J. Infect. Dis. 2011, 203, 1753-1762. [CrossRef]

6. Huang, J.; Yu, X.; Fries, J.W.; Zhang, L.; Odenthal, M. MicroRNA function in the profibrogenic interplay upon chronic liver disease. Int. J. Mol. Sci. 2014, 15, 9360-9371. [CrossRef]

7. Shpilka, T.; Haynes, C.M. The mitochondrial UPR: Mechanisms, physiological functions and implications in ageing. Nat. Rev. Mol. Cell Biol. 2018, 19, 109-120. [CrossRef]

8. Yi, H.S. Implications of Mitochondrial Unfolded Protein Response and Mitokines: A Perspective on Fatty Liver Diseases. Endocrinol. Metab. 2019, 34, 39-46. [CrossRef] 
9. Liu, R.; Li, X.; Huang, Z.; Zhao, D.; Ganesh, B.S.; Lai, G.; Pandak, W.M.; Hylemon, P.B.; Bajaj, J.S.; Sanyal, A.J.; et al. C/EBP homologous protein-induced loss of intestinal epithelial stemness contributes to bile duct ligation-induced cholestatic liver injury in mice. Hepatology 2018, 67, 1441-1457. [CrossRef]

10. Gonzalez-Rodriguez, A.; Mayoral, R.; Agra, N.; Valdecantos, M.P.; Pardo, V.; Miquilena-Colina, M.E.; Vargas-Castrillon, J.; Lo Iacono, O.; Corazzari, M.; Fimia, G.M.; et al. Impaired autophagic flux is associated with increased endoplasmic reticulum stress during the development of NAFLD. Cell Death Dis. 2014, 5, e1179. [CrossRef]

11. Winnay, J.N.; Boucher, J.; Mori, M.A.; Ueki, K.; Kahn, C.R. A regulatory subunit of phosphoinositide 3-kinase increases the nuclear accumulation of X-box-binding protein-1 to modulate the unfolded protein response. Nat. Med. 2010, 16, 438-445. [CrossRef]

12. Chen, X.; Bian, M.; Zhang, C.; Kai, J.; Yao, Z.; Jin, H.; Lu, C.; Shao, J.; Chen, A.; Zhang, F.; et al. Dihydroartemisinin inhibits ER stress-mediated mitochondrial pathway to attenuate hepatocyte lipoapoptosis via blocking the activation of the PI3K/Akt pathway. Biomed. Pharmacother. 2018, 97, 975-984. [CrossRef] [PubMed]

13. Yang, Y.L.; Kuo, H.C.; Wang, F.S.; Huang, Y.H. MicroRNA-29a Disrupts DNMT3b to Ameliorate Diet-Induced Non-Alcoholic Steatohepatitis in Mice. Int. J. Mol. Sci. 2019, 20, 1499. [CrossRef] [PubMed]

14. Lin, H.Y.; Wang, F.S.; Yang, Y.L.; Huang, Y.H. MicroRNA-29a Suppresses CD36 to Ameliorate High Fat Diet-Induced Steatohepatitis and Liver Fibrosis in Mice. Cells 2019, 8, 1298. [CrossRef]

15. Huang, Y.H.; Kuo, H.C.; Yang, Y.L.; Wang, F.S. MicroRNA-29a is a key regulon that regulates BRD4 and mitigates liver fibrosis in mice by inhibiting hepatic stellate cell activation. Int. J. Med. Sci. 2019, 16, $212-220$. [CrossRef]

16. Lin, Y.C.; Wang, F.S.; Yang, Y.L.; Chuang, Y.T.; Huang, Y.H. MicroRNA-29a mitigation of toll-like receptor 2 and 4 signaling and alleviation of obstructive jaundice-induced fibrosis in mice. Biochem. Biophys. Res. Commun. 2018, 496, 880-886. [CrossRef]

17. Huang, Y.H.; Yang, Y.L.; Wang, F.S. The Role of miR-29a in the Regulation, Function, and Signaling of Liver Fibrosis. Int. J. Mol. Sci. 2018, 19, 1889. [CrossRef]

18. Huang, Y.H.; Yang, Y.L.; Huang, F.C.; Tiao, M.M.; Lin, Y.C.; Tsai, M.H.; Wang, F.S. MicroRNA-29a mitigation of endoplasmic reticulum and autophagy aberrance counteracts in obstructive jaundice-induced fibrosis in mice. Exp. Biol. Med. 2018, 243, 13-21. [CrossRef]

19. Yang, Y.L.; Wang, F.S.; Li, S.C.; Tiao, M.M.; Huang, Y.H. MicroRNA-29a Alleviates Bile Duct Ligation Exacerbation of Hepatic Fibrosis in Mice through Epigenetic Control of Methyltransferases. Int. J. Mol. Sci. 2017, 18, 192. [CrossRef]

20. Li, S.C.; Wang, F.S.; Yang, Y.L.; Tiao, M.M.; Chuang, J.H.; Huang, Y.H. Microarray Study of Pathway Analysis Expression Profile Associated with MicroRNA-29a with Regard to Murine Cholestatic Liver Injuries. Int. J. Mol. Sci. 2016, 17, 324. [CrossRef]

21. Huang, Y.H.; Tiao, M.M.; Huang, L.T.; Chuang, J.H.; Kuo, K.C.; Yang, Y.L.; Wang, F.S. Activation of Mir-29a in Activated Hepatic Stellate Cells Modulates Its Profibrogenic Phenotype through Inhibition of Histone Deacetylases 4. PLoS ONE 2015, 10, e0136453. [CrossRef] [PubMed]

22. Jackson, A.L.; Linsley, P.S. Recognizing and avoiding siRNA off-target effects for target identification and therapeutic application. Nat. Rev. Drug Discov. 2010, 9, 57-67. [CrossRef] [PubMed]

23. Matsumoto, Y.; Itami, S.; Kuroda, M.; Yoshizato, K.; Kawada, N.; Murakami, Y. MiR-29a Assists in Preventing the Activation of Human Stellate Cells and Promotes Recovery From Liver Fibrosis in Mice. Mol. Ther. 2016, 24, 1848-1859. [CrossRef] [PubMed]

24. Chu, Q.; Martinez, T.F.; Novak, S.W.; Donaldson, C.J.; Tan, D.; Vaughan, J.M.; Chang, T.; Diedrich, J.K.; Andrade, L.; Kim, A.; et al. Regulation of the ER stress response by a mitochondrial microprotein. Nat. Commun. 2019, 10, 4883. [CrossRef]

25. Senft, D.; Ronai, Z.A. UPR, autophagy, and mitochondria crosstalk underlies the ER stress response. Trends Biochem. Sci. 2015, 40, 141-148. [CrossRef]

26. Gore, E.; Bigaeva, E.; Oldenburger, A.; Kim, Y.O.; Rippmann, J.F.; Schuppan, D.; Boersema, M.; Olinga, P. PI3K inhibition reduces murine and human liver fibrogenesis in precision-cut liver slices. Biochem. Pharmacol. 2019, 169, 113633. [CrossRef] 
27. Son, M.K.; Ryu, Y.L.; Jung, K.H.; Lee, H.; Lee, H.S.; Yan, H.H.; Park, H.J.; Ryu, J.K.; Suh, J.K.; Hong, S.; et al. HS-173, a novel PI3K inhibitor, attenuates the activation of hepatic stellate cells in liver fibrosis. Sci. Rep. 2013, 3, 3470. [CrossRef]

28. Son, G.; Hines, I.N.; Lindquist, J.; Schrum, L.W.; Rippe, R.A. Inhibition of phosphatidylinositol 3-kinase signaling in hepatic stellate cells blocks the progression of hepatic fibrosis. Hepatology 2009, 50, 1512-1523. [CrossRef]

29. Liu, W.; Jing, Z.T.; Xue, C.R.; Wu, S.X.; Chen, W.N.; Lin, X.J.; Lin, X. PI3K/AKT inhibitors aggravate death receptor-mediated hepatocyte apoptosis and liver injury. Toxicol. Appl. Pharmacol. 2019, 381, 114729. [CrossRef]

30. Duan, M.; Yang, Y.; Peng, S.; Liu, X.; Zhong, J.; Guo, Y.; Lu, M.; Nie, H.; Ren, B.; Zhang, X.; et al. C/EBP Homologous Protein (CHOP) Activates Macrophages and Promotes Liver Fibrosis in Schistosoma japonicum-Infected Mice. J. Immunol. Res. 2019, 2019, 5148575. [CrossRef]

31. Zhang, R.; Wang, X.; Qu, J.H.; Liu, B.; Zhang, P.; Zhang, T.; Fan, P.C.; Wang, X.M.; Xiao, G.Y.; Su, Y.; et al. Caloric Restriction Induces MicroRNAs to Improve Mitochondrial Proteostasis. Science 2019, 17, 155-166. [CrossRef] [PubMed]

(C) 2020 by the authors. Licensee MDPI, Basel, Switzerland. This article is an open access article distributed under the terms and conditions of the Creative Commons Attribution (CC BY) license (http://creativecommons.org/licenses/by/4.0/). 